\title{
Annihilators in JB-algebras
}

\author{
By M. BATTAGLIA \\ Universität Bern, Sidlerstrasse 5, CH-3012 Bern, Switzerland
}

(Received 9 October 1989; revised 11 January 1990)

\begin{abstract}
Orthogonality is defined for all elements in a JB-algebra and Topping's results on annihilators in $\mathrm{JW}$-algebras are generalized to the context of JB-and JBW-algebras. A pair $(a, b)$ of elements in a JB-algebra $A$ is said to be orthogonal provided that $a^{2} \circ b$ equals zero. It is shown that this relation is symmetric. The annihilator $S^{\perp}$ of a subset $S$ of $A$ is defined to be the set of elements $a$ in $A$ such that, for all elements $s$ in $S$, the pair $(s, a)$ is orthogonal. It is shown that the annihilators are closed quadratic ideals and, if $A$ is a JBW-algebra, a subset $I$ of $A$ is a w*-closed quadratic ideal if and only if $I$ coincides with its biannihilator $I^{\perp \perp}$. Moreover, in a JBW-algebra $A$ the formation of the annihilator of a $w^{*}$-closed quadratic ideal is an orthocomplementation on the complete lattice of $w^{*}$-closed quadratic ideals which makes it into a complete orthomodular lattice. Further results establish a connection between ideals, central idempotents and annihilators in JBW-algebras.
\end{abstract}

\section{Introduction}

Topping[7] studied the $w^{*}$-closed Jordan algebras of bounded self-adjoint operators, the so-called JW-algebras. A generalization of these objects was given by Alfsen, Shultz and Størmer[1] by introducing JB-and JBW-algebras.

It is the aim of the present paper to generalize Topping's theory on annihilators in $\mathrm{JW}$-algebras to the context of JB- and JBW-algebras. Annihilators in JWalgebras are defined in terms of the underlying associative operator product and therefore there is no obvious extension of this definition to JB-algebras.

Let $A$ be a JB-algebra. A pair $(a, b)$ of elements in $A$ is said to be orthogonal provided that $a^{2} \circ b$ equals zero. The relation of orthogonality is symmetric: this is shown in Section 3.

In Section 4 the annihilator $S^{\perp}$ of a subset $S$ of the JB-algebra $A$ is defined as the set of elements $a$ in $A$ such that, for all elements $s$ in $S$, the pair $(s, a)$ is orthogonal. It is shown that the annihilator of a general subset $S$ of a JB-algebra $A$ is a closed quadratic ideal.

In Section 5 it is shown that a subset $I$ of a JBW-algebra $A$ is a w $^{*}$-closed quadratic ideal if and only if $I$ coincides with its biannihilator $I^{\perp \perp}$. It also follows that the formation of the annihilator of a $w^{*}$-closed quadratic ideal in a JBW-algebra $A$ is an orthocomplementation on the complete lattice of $w^{*}$-closed quadratic ideals which makes it into a complete orthomodular lattice. Further results establish a connection between ideals, central idempotents and annihilators in JBW-algebras. 


\section{Preliminaries}

A (real) Jordan algebra $A$ is a real vector space together with a bilinear mapping $\circ: A^{2} \rightarrow A$, called the Jordan product, satisfying, for all elements $a$ and $b$ in $A$,

$$
\text { (i) } a \circ b=b \circ a, \text { (ii) }\left(a^{2} \circ b\right) \circ a=a^{2} \circ(b \circ a) \text {. }
$$

In a real associative algebra $(A,$.$) a canonical Jordan product can be defined, for all$ elements $a$ and $b$ in $A$, by

$$
a \circ b:=\frac{1}{2}(a \cdot b+b \cdot a)
$$

A Jordan algebra which is isomorphic to a subalgebra of a Jordan algebra formed in this way is said to be special.

Let $A$ be a Jordan algebra and let $a$ be an element in $A$. Then a linear mapping $U_{a}: A \rightarrow A$ is defined, for all elements $b$ in $A$, by

$$
U_{a} b:=2(a \circ b) \circ a-a^{2} \circ b .
$$

A subspace $I$ of a Jordan algebra $A$ is said to be an ideal, resp. a quadratic ideal, provided that $I \circ A \subseteq I$, resp. $U_{I} A \subseteq I$. An element $a$ in a Jordan algebra $A$ is called central provided that, for all elements $b$ and $c$ in $A,(a \circ b) \circ c$ equals $a \circ(b \circ c)$. The set $Z(A)$ of all central elements in $A$ is called the centre of $A$. Notice that $Z(A)$ is a subalgebra.

A Jordan algebra $A$ with unit $e$ together with a complete norm $\|\cdot\|$ such that, for all elements $a$ and $b$ in $A$,

$$
\text { (i) }\|a \circ b\| \leqslant\|a\|\|b\|, \text { (ii) }\|a\|^{2} \leqslant\left\|a^{2}+b^{2}\right\|
$$

is called a $J B$-algebra. Notice that in a JB-algebra the Jordan product is jointly continuous. The set $A^{2}$ of all squares of elements in a JB-algebra $A$ is a proper convex cone. Therefore $A^{2}$ induces an ordering $\leqslant$ on $A$. For all elements $a$ in a JB-algebra $A$, the mapping $U_{a}: A \rightarrow A$ is positive, i.e. $U_{a} A^{2} \subseteq A^{2}$.

A JB-algebra $A$ which is a Banach space dual is said to be a $J B W$-algebra. Notice that in a $\mathrm{JBW}$-algebra the Jordan product is separately $\mathrm{w}^{*}$-continuous and that a $\mathrm{w}^{*}$-closed subalgebra of a JBW-algebra has a unit.

An element $p$ in a JB-algebra $A$ is called idempotent if $p^{2}=p$. Let $U(A)$ denote the collection of idempotents in $A$. With respect to the ordering $\leqslant$ and the orthocomplementation defined, for all idempotents $p$ in $A$, by $p^{\prime}:=e-p, U(A)$ becomes an orthomodular poset with 0 as the least and $e$ as the greatest element. Let $p$ and $q$ be idempotents in $A$. Then $p \leqslant q^{\prime}$ if and only if $p \circ q$ equals zero and $p \leqslant q$ if and only if $p \circ q$ equals $p$ if and only if $U_{q} U_{p}$ equals $U_{p}$. In a JBW-algebra $A$ the triple $\left(U(A), \leqslant,^{\prime}\right)$ is a complete orthomodular lattice.

Let $a$ be an element in a JBW-algebra $A$. Then there exists a least idempotent $r(a)$ in $A$ such that $r(a) \circ a$ equals $a$. The element $r(a)$ is called the support idempotent of $a$. Notice that $r(a)$ is the unit in the smallest $\mathrm{w}^{*}$-closed subalgebra $W(a)$ of $A$ containing $a$. For every idempotent $p$ in a JBW-algebra $A$ there exists a least central idempotent $c(p)$ in $A$ majorizing $p$. The element $c(p)$ is called the central support of $p$.

Edwards [2] has shown that every $w^{*}$-closed quadratic ideal in a JBW-algebra $A$ is of the form $U_{p} A$, for a suitable idempotent $p$ in $A$, and conversely, for an 
idempotent $p$ in $A, U_{p} A$ is a $\mathrm{w}^{*}$-closed quadratic ideal. Moreover, $U_{p} A$ is an ideal if and only if $p$ is central.

For the details of the theory of JB-and JBW-algebras the reader is referred to $[1,2,3,4,6]$.

\section{Orthogonality in $J B$-algebras}

Let $A$ be a JB-algebra. A pair $(a, b)$ of elements in $A$ is said to be orthogonal, denoted by $a \perp b$, if $a^{2} \circ b=0$. Hence a pair $(p, q)$ of idempotents is orthogonal if and only if $p \leqslant q^{\prime}$.

Notice that, for elements $a$ and $b$ in a JB-algebra $A, a \circ b=0$ does not imply $a^{2} \circ b=0$. In the JB-algebra $M_{3}^{8}$, i.e. the Jordan algebra of hermitian $3 \times 3$ matrices with Cayley number entries, consider the elements

$$
a=\left(\begin{array}{ccc}
0 & i & 0 \\
-i & 0 & -i \\
0 & i & 0
\end{array}\right), \quad b=\left(\begin{array}{ccc}
0 & -k & 0 \\
k & 0 & k \\
0 & -k & 0
\end{array}\right)
$$

Since $i k=-j, k i=j$ and $i^{2}=-1, a \circ b=0$ but $a^{2} \circ b=2 b \neq 0$.

It is the aim of this section to show that orthogonality is a symmetric relation and that $a \perp b$ entails $a^{n} \circ b=0$ for all natural numbers $n$.

Lемма 3-1. Let $A$ be a JB-algebra. Let $(a, b)$ be an orthogonal pair of elements in $A$. Then $a^{2} \circ b^{2}$ is a positive element in $A$.

Proof. By [5], theorem $\mathrm{I} \cdot 10$, the smallest subalgebra $B$ of $A$ containing $a^{2}$ and $b$ is special, i.e. there is an isomorphism $j$ from $B$ into an associative algebra $(J,$. equipped with the canonical Jordan product. Then

$$
j(a)^{2} \cdot j(b)+j(b) \cdot j(a)^{2}=0
$$

Multiplying the equation on the left and right, respectively, by $j(b)$ in the underlying associative product we obtain

$$
j(a)^{2} \cdot j(b)^{2}=j(b)^{2} \cdot j(a)^{2} .
$$

Therefore $a^{2}$ and $b^{2}$ generate an associative subalgebra in $A$. By the continuity of the Jordan product, the smallest closed subalgebra $C$ of $A$ containing $a^{2}$ and $b^{2}$ is associative as well. Since $a^{2}$ and $b^{2}$ are positive elements in $A$, there exist, by [2], lemma $2 \cdot 1$, elements $c$ and $d$ in $C$ such that $a^{2}=c^{2}$ and $b^{2}=d^{2}$. By the associativity of $C$, we have $(c \circ d)^{2}=a^{2} \circ b^{2}$.

Lemмa $3 \cdot 2$. Let $A$ be a JB-algebra. Let $(a, b)$ be an orthogonal pair of elements in $A$. Then

$$
a^{2} \circ b^{2}=U_{b} a^{2}=U_{a} b^{2}=U_{a} b=a \circ b=0 .
$$

Proof. From $a \perp b$ it follows that $U_{b} a^{2}=-a^{2} \circ b^{2}$. Then, by Lemma $3 \cdot 1$ and the positivity of the operator $U_{b}$, both $U_{b} a^{2}$ and $a^{2} \circ b^{2}$ are zero. The remaining assertions follow from the identities

$$
\begin{gathered}
\left(U_{a} b^{2}\right)^{2}=U_{a} U_{b} U_{b} a^{2}, \quad\left(U_{a} b\right)^{2}=U_{a} U_{b} a^{2}, \\
(a \circ b)^{2}=\frac{1}{2} b \circ U_{a} b+\frac{1}{4} U_{a} b^{2}+\frac{1}{4} U_{b} a^{2} .
\end{gathered}
$$


Corollary $3 \cdot 3$. Let $A$ be $a$ JB-algebra and let $a$ and $b$ be elements in $A$. Then the following conditions are equivalent:

(i) $a \perp b ; \quad$ (ii) $a \circ b=0$ and $a^{2} \circ b=0$; (iii) $a^{n} \circ b=0$ for all $n \in \mathbb{N}$.

Proof. (i) $\Rightarrow$ (ii). See Lemma $3 \cdot 2$.

(ii) $\Rightarrow$ (iii). By [5], theorem I·10, the smallest subalgebra $B$ of $A$ containing $a$ and $b$ is special, i.e. there is an isomorphism $j$ from $B$ into an associative algebra $(J,$. equipped with the canonical Jordan product. Suppose now that $a^{n-1} \circ b=0$. Then

$$
j(a)^{n-1} \cdot j(b)+j(b) \cdot j(a)^{n-1}=0 .
$$

Multiplying the equation on the left and right, respectively, by $j(a)$ in the underlying asociative product and adding the results we get

$$
j(a)^{n} \cdot j(b)+j(b) \cdot j(a)^{n}+j(a)^{n-1} \cdot j(b) \cdot j(a)+j(a) \cdot j(b) \cdot j(a)^{n-1}=0 .
$$

From this and the assumption it follows that $a^{n} \circ b=0$.

(iii) $\Rightarrow$ (i). This is obvious.

We now proceed to the main result of this section.

Proposition 3*4. Let $A$ be $a J B$-algebra. Let $a$ and $b$ be elements in $A$. Then $a \perp b$ if and only if $b \perp a$.

Proof. Suppose that $a \perp b$. By Lemma $3 \cdot 2$ and the positivity of $U_{a}$ and $U_{b}$,

$$
0 \leqslant U_{a} b^{4} \leqslant\left\|b^{2}\right\| U_{a} b^{2}=0 .
$$

Therefore $U_{a} b^{4}$ equals zero and the assertion now follows from Lemma $3 \cdot 2$ and the identity

$$
\left(a \circ b^{2}\right)^{2}=\frac{1}{4} U_{a} b^{4}+\frac{1}{4} U_{b^{2}} a^{2}+\frac{1}{2} b^{2} \circ U_{a} b^{2} .
$$

\section{Annihilators in JB-algebras}

Let $A$ be a JB-algebra and let $S$ be a subset of $A$. The annihilator $S^{\perp}$ of $S$ is defined to be

$$
S^{\perp}:=\{a \in A ; s \perp a \quad \text { for all } s \in S\} .
$$

The set $S^{\perp \perp}$ is referred to as the biannihilator of $S$.

Lemma 4.1. Let $A$ be a JB-algebra, let $S$ and $T$ be subsets of $A$ and let $\left(S_{i}\right)_{i \in I}$ be a family of subsets of $A$. Then

(i) $S \subseteq T$ implies $T^{\perp} \subseteq S^{\perp}$;

(iii) $\left(\bigcup_{i \in I} S_{i}\right)^{\perp}=\bigcap_{i \in I}\left(S_{i}\right)^{\perp}$;

(ii) $S \subseteq S^{\perp \perp}$;

(v) $S^{\perp}$ is a closed subspace of $A$;

(iv) $S^{\perp \perp \perp}=S^{\perp}$;

(vi) $S^{\perp}=(\operatorname{cl} S)^{\perp}=(\operatorname{lin} S)^{\perp}$.

Proof. (i), (ii), (iii) and (iv). These are immediate.

(v) By bilinearity and continuity of the Jordan product, $S^{\perp}$ is a closed subspace.

(vi) By (v), cl $S$ and lin $S$ all are contained in $S^{\perp \perp}$ and the assertion follows, by (i).

Theorem 4.2. Let $A$ be a JB-algebra and let $S$ be a subset of $A$. Then the annihilator $S^{\perp}$ of $S$ is a closed quadratic ideal. 
Proof. Let $a, b$ and $c$ be elements in $A$ such that the pair $(a, b)$ is orthogonal. By the positivity of the operators $U_{a^{2}}, U_{b}$ and $U_{c}$ and Lemma $3 \cdot 2$, it follows that

$$
0 \leqslant U_{a^{2}} U_{b} U_{c} b^{2} \leqslant\left\|b^{2}\right\| U_{a^{2}} U_{b} c^{2} \leqslant\left\|b^{2}\right\|\left\|c^{2}\right\| U_{a^{2}} b^{2}=0 .
$$

Therefore $U_{a^{2}} U_{b} U_{c} b^{2}=0$. Since $0 \leqslant a^{4} \leqslant\left\|a^{2}\right\| a^{2}$, we conclude, by Lemma $3 \cdot 2$, that

$$
0 \leqslant U_{b} a^{4} \leqslant\left\|a^{2}\right\| U_{b} a^{2}=0 .
$$

Therefore $U_{b} a^{4}=0$. By Lemma $3 \cdot 2$ and the identity

$$
\left(a^{2} \circ U_{b} c\right)^{2}=\frac{1}{4} U_{a^{2}} U_{b} U_{c} b^{2}+\frac{1}{4} U_{b} U_{c} U_{b} a^{4}+\frac{1}{2} a^{2} \circ U_{b} U_{c} U_{b} a^{2},
$$

$a^{2} \circ U_{b} c$ equals zero. Therefore by Lemma $4 \cdot 1(\mathrm{v})$, the assertion follows.

Proposition 4.3. Let $A$ be a JB-algebra and let $p$ and $q$ be idempotents in $A$. Then

(i) $\{p\}^{\perp}=U_{p^{\prime}} A=\left(U_{p} A\right)^{\perp}$, and (ii) $p \leqslant q$ if and only if $\{p\}^{\perp} \supseteq\{q\}^{\perp}$.

Proof. (i) Let $a$ be an element in $U_{p} A$ and let $b$ be an element in $U_{p^{\prime}} A$. Then, by (2.66) and (2.69) from [4], we have $a^{2} \circ b=0$. Therefore $U_{p^{\prime}} A \subseteq\left(U_{p} A\right)^{\perp}$. By Lemma 4.1 (i), we have $\left(U_{p} A\right)^{\perp} \subseteq\{p\}^{\perp}$. Now let $b \in\{p\}^{\perp}$; then $p^{\prime} \circ b=b$. Therefore $b \in U_{p^{\prime}} A$.

(ii) Since $p \leqslant q$ if and only if $q^{\prime} \leqslant p^{\prime}$, (ii) follows from (i) and $U_{q^{\prime}} A \subseteq U_{p^{\prime}} A$.

\section{Annihilators in $J B W$-algebras}

In JBW-algebras Lemma $4 \cdot 1$ (vi) and Theorem $4 \cdot 2$ can be improved.

Lemma 5-1. Let $A$ be a JBW-algebra and let $S$ be a subset of $A$. Then

(i) $S^{\perp}$ is a $\mathrm{w}^{*}$-closed quadratic ideal in $A$, and

(ii) $S^{\perp}=(\operatorname{cl} S)^{\perp}=\left(w^{*}-\operatorname{cl} S\right)^{\perp}=(\operatorname{lin} S)^{\perp}$.

Proof. By Theorem $4 \cdot 2$ and separate $w^{*}$-continuity of the Jordan product, (i) follows. The proof of (ii) is similar to that of Lemma $4 \cdot 1$ (vi).

We are now in a position to identify the subsets $S$ of a JBW-algebra which coincide with their biannihilator $S^{\perp \perp}$.

Theorem 5.2. Let $A$ be a JBW-algebra. A subset $S$ of $A$ is a $\mathrm{w}^{*}$-closed quadratic ideal if and only if $S$ coincides with its biannihilator $S^{\perp \perp}$.

Proof. By Lemma 5.1, a subset which coincides with its biannihilator is a $w^{*}$-closed quadratic ideal. Since every $\mathrm{w}^{*}$-closed quadratic ideal is of the form $U_{p} A$ for a suitable idempotent $p$ in $A$, by [2], theorem 233, it follows from Proposition 4.3 that it coincides with its biannihilator.

Corollary 5:3. Let $A$ be a JBW-algebra, let $U(A)$ be the collection of idempotents in $A$ and let $\mathscr{T}(A)$ be the collection of $\mathrm{w}^{*}$-closed quadratic ideals in $A$. Let $p$ be an idempotent in $A$ and define $\phi(p):=U_{p} A$. Then the mapping $\phi: U(A) \rightarrow \mathscr{T}(A)$ is an ortho-order isomorphism from the complete orthomodular lattice $\left(U(A), \leqslant,^{\prime}\right)$ onto $\left(\mathscr{T}(A), \subseteq,{ }^{\perp}\right)$.

Proof. By Theorem 5.2, Proposition $4 \cdot 3$ and [2], corollary 2.5, the assertion follows.

In order to identify the annihilator of a general subset of a JBW-algebra we begin by examining annihilators of singleton sets.

Lemma 5.4. Let $A$ be a JBW-algebra and let $a$ and $b$ be elements in $A$. Then the following conditions are pairwise equivalent: (i) $a \perp b$; (ii) $a \perp r(b)$; (iii) $r(a) \perp r(b)$. 
Proof. (i) $\Rightarrow$ (ii) $\Rightarrow$ (iii). Since $W(b)$ coincides with the $w^{*}$-closure of the linear hull of the set $\left\{b^{n} ; n \in \mathbb{N}\right\}$ and since the Jordan product is separately $w^{*}$-continuous, this follows from Corollary $3 \cdot 3$.

(iii) $\Rightarrow$ (i). If $r(a) \circ r(b)$ equals zero then $r(a) \leqslant r(b)^{\prime}$. Therefore $p \leqslant r(b)^{\prime}$ for all idempotents $p$ in $W(a)$, and it follows that $r(b) \leqslant p^{\prime}$. Hence $p \circ q=0$ for all idempotents $q$ in $W(b)$ and all idempotents $p$ in $W(a)$, and, by spectral theory, $a \perp b$.

Corollary 5.5. Let $A$ be a JBW-algebra and let a be an element in $A$. Then $r(a)$ is the unique idempotent in $A$ such that $\{a\}^{\perp}=\{r(a)\}^{\perp}$.

Proof. By Lemma 5*4, $a \perp b$ if and only if $r(a) \perp b$. Hence $\{a\}^{\perp}=\{r(a)\}^{\perp}$. Uniqueness follows from Corollary $5 \cdot 3$.

TheOREM 5.6. Let $A$ be a $J B W$-algebra and let $S$ be a non-empty subset of $A$. Let $W(S)$ denote the smallest $\mathrm{w}^{*}$-closed subalgebra of $A$ containing $S$ and let $p$ be the unit of $W(S)$. Then $S^{\perp}=U_{p^{\prime}} A$.

Proof. By Lemma $4 \cdot 1$ (iii), Corollary 5.5, Proposition $4 \cdot 3$ and Corollary 5.3,

$$
S^{\perp}=\bigcap_{a \in S}\{a\}^{\perp}=\bigcap_{a \in S}\{r(a)\}^{\perp}=\bigcap_{a \in S} U_{r(a)^{\prime}} A=U_{r^{\prime}} A,
$$

where $r$ denotes $\bigvee_{a \in S} r(a)$. It remains to prove that $r=p$. Clearly $r \leqslant p$. Conversely, $r(a) \leqslant r$, for all elements $a$ in $S$. Therefore $W(S) \subseteq U_{r} A$ and $p \leqslant r$ follows.

We present two applications of the previously developed theory.

Corollary 5-7. Let $A$ be a JBW-algebra, let $S$ be a subset of $A$ and let $W(S)$ denote the smallest $\mathrm{w}^{*}$-closed subalgebra of $A$ containing $S$. Then

$$
S^{\perp}=\{a \in A ; W(S) \circ a=\{0\}\}
$$

Proof. Let $p$ be the unit of $W(S)$. Then $W(S) \subseteq U_{p} A$ and, by Theorem $5 \cdot 6, S^{\perp}$ equals $U_{p^{\prime}} A$. Hence, by [4], theorem $2 \cdot 6 \cdot 5$, we have $W(S) \circ S^{\perp}=\{0\}$. Conversely, if $W(S) \circ a=\{0\}$ then $b^{2} \circ a=0$ for all $b$ in $S$.

Corollary 5-8. Let $A$ be a JBW-algebra and let $a$ and $b$ be elements in $A$. Then $r(a+b) \leqslant r(a) \vee r(b)$.

Proof. From $r(a) \leqslant r(a) \vee r(b), r(b) \leqslant r(a) \vee r(b)$, Proposition 4.3 and Corollary 5.5 it follows that $\{r(a) \vee r(b)\}^{\perp} \subseteq\{a\}^{\perp} \cap\{b\}^{\perp}$. Therefore, for all $z$ in $\{r(a) \vee r(b)\}^{\perp}$, both $a \circ z^{2}$ and $b \circ z^{2}$ equal zero. Hence $(a+b) \circ z^{2}=0$ and

$$
z \in\{a+b\}^{\perp}=\{r(a+b)\}^{\perp}
$$

by Corollary 5.5. Therefore $\{r(a) \vee r(b)\}^{\perp} \subseteq\{r(a+b)\}^{\perp}$ and an application of Proposition $4 \cdot 3$ proves the claim.

The final results generalize [7], proposition 5 and section 7 .

Proposition 59. Let $A$ be a JBW-algebra and let $I$ be an ideal in $A$. Then there exists a central idempotent $f$ in $A$ such that $I^{\perp}=U_{f} A$.

Proof. By the separate $\mathrm{w}^{*}$-continuity of the Jordan product, $\mathrm{w}^{*}$-cl $I$ is an ideal. By [2], theorem 2:3, there is a central idempotent $f$ in $A$ such that $\mathrm{w}^{*}$-cl $I=U_{f} A$. Then $f^{\prime}$ is a central idempotent in $A$ and $I^{\perp}=U_{f^{\prime}} A$, by Theorem 5.6 and Lemma 5.1. 
Proposition 5·10. Let $A$ be a JBW-algebra and let a be an element in $A$. Let (a) be the smallest ideal containing $a$ and let $r(a)$ be the support idempotent of $a$. Then the central support $c(r(a))$ of $r(a)$ is the unique (central) idempotent such that $\{c(r(a))\}^{\perp}=(a)^{\perp}$.

Proof. By Proposition 5.9, there is a central idempotent $f$ in $A$ such that

$$
(a)^{\perp}=\{f\}^{\perp}=U_{f^{\prime}} A \text {. }
$$

Hence $f \circ a=a$. Therefore $c(r(a)) \leqslant f$. Since $U_{c(r(a))} A$ is an ideal containing $a$, it follows that

$$
U_{c(r(a))^{\prime}} A=\left(U_{c(r(a))} A\right)^{\perp} \subseteq(a)^{\perp}=U_{f} A .
$$

Therefore $c(r(a))^{\prime} \leqslant f^{\prime}$.

Corollary 5-11. Let $A$ be a JBW-algebra and let $p$ and $q$ be idempotents in $A$. Let $c(p)$ and $c(q)$ be the central supports of $p$ and $q$, respectively. Let $(p)$ and $(q)$ be the smallest ideals containing $p$ and $q$, respectively. Then the following conditions are equivalent:

(i) $c(p) \perp c(q)$; (ii) $c(p) \perp q$; (iii) $p \perp c(q)$; (iv) $q \in(p)^{\perp}$; (v) $p \in(q)^{\perp}$.

Proof. (i) $\Leftrightarrow$ (ii) $\Leftrightarrow$ (iii). These are immediate.

(ii) $\Leftrightarrow$ (iv). Since $U_{c(p)} A$ is an ideal containing $p, c(p) \perp q$ entails

$$
q \in U_{c(p)} A=\left(U_{c(p)} A\right)^{\perp} \subseteq(p)^{\perp} .
$$

Conversely, let $q$ be an element in $(p)^{\perp}$. Then, by Proposition 5·10, $q$ is contained in $\{c(p)\}^{\perp}$. Therefore $c(p) \perp q$.

(iii) $\Leftrightarrow(v)$. The proof is similar to the one above.

Proposition 5·12. Let $A$ be a $J B W$-algebra with centre $Z(A)$ and let $S$ be a subset of $Z(A)$. Then $S^{\perp}$ is a $\mathrm{w}^{*}$-closed ideal in $A$.

Proof. By Lemma $5 \cdot 1, S^{\perp}$ is a w*-closed subspace of $A$. Let $a \in A$ and $b \in S^{\perp}$. It is enough to show $c \perp a \circ b$, for each element $c$ in $S$. Since $c$ is central, $c^{2}$ also is central and it follows that

$$
c^{2} \circ(b \circ a)=\left(c^{2} \circ b\right) \circ a=0 .
$$

The author wishes to thank G. T. Rüttimann for encouragement and valuable discussions and the Swiss National Science Foundation for supporting this research.

\section{REFERENCES}

[1] E. M. Alfsen, F. W. Schultz and E. Størmer. A Gelfand-Neumark theorem for Jordan algebras. Adv. in Math. 28 (1978), 11-56.

[2] C. M. Edwards. Ideal theory in JB-Algebras. J. London Math. Soc. (2) 16 (1977), 507 513.

[3] C. M. Edwards. On the facial structure of a JB-algebra. J. London Math. Soc. (2) 19 (1979), 335-344.

[4] H. Hanche-Olsen and E. Størmer. Jordan Operator Algebras. Monographs Stud. Math. no. 21 (Pitman, 1984).

[5] N. JacoBson. Structure and representations of Jordan algebras. Amer. Math. Soc. Colloq. Publ. no. 39 (American Mathematical Society, 1968).

[6] F. W. Shcltz. On normed Jordan algebras which are Banach dual spaces. J. Funct. Anal. 31 (1979), 360-376.

[7] D. M. ToppING. Jordan algebras of self-adjoint operators. Memoirs Amer. Math. Soc. no. 53 (American Mathematical Society, 1965). 